\title{
NOTE ON ROTATION SET
}

\author{
RYUICHI ITO
}

\begin{abstract}
Let $f$ be an endomorphism of the circle of degree 1 and $\bar{f}$ be a lifting of $f$. We characterize the rotation set $\rho(\bar{f})$ by the set of probability measures on the circle, and prove that if $\rho_{+}(\bar{f})\left(\rho_{-}(\bar{f})\right)$, the upper (lower) endpoint of $\rho(\bar{f})$, is irrational, then $\rho_{+}\left(R_{\theta} \bar{f}\right)>\rho_{+}(\bar{f})\left(\rho_{-}\left(R_{\theta} \bar{f}\right)>\rho_{-}(\bar{f})\right)$ for any $\theta>0$, where $R_{\theta}(x)$ $=x+\theta$. As a corollary, if $f$ is structurally stable, then both $\rho_{+}(\bar{f})$ and $\rho_{-}(\bar{f})$ are rational.
\end{abstract}

Newhouse, Palis and Takens [3] have generalized a rotation number for a homeomorphism of the circle to a continuous map of degree 1 and defined a rotation set. Let $R$ denote the real numbers, $Z$ the integers, $N$ the positive integers and $S=R / Z$ the circle. Let $\pi: R \rightarrow S$ denote the canonical projection. Let $f: S \rightarrow S$ be a given continuous map of degree 1 . Choose a lifting $\bar{f}: R \rightarrow R$, that is a map such that $\pi \bar{f}=f \pi$. Liftings exist and are unique up to the addition of an integer. Each lifting satisfies $\bar{f}(x+1)=\bar{f}(x)+1$.

Definition. Given $x \in R$, define the rotation number

$$
\rho(\bar{f}, x)=\limsup _{n \rightarrow \infty} \frac{1}{n}\left(\bar{f}^{n}(x)-x\right) .
$$

Define the rotation set to be $\rho(\bar{f})=\{\rho(\bar{f}, x) \mid x \in R\}$.

Notice that if a different lifting is used, this mearly has the effect of translating the rotation set by an integer.

We recall the following properties of $\rho(\bar{f})$.

(1) If $f=h g h^{-1}$ for an orientation preserving homeomorphism $h$ of $S$, then $\rho(\bar{f})=\rho(\bar{g})$ for suitable liftings $\bar{f}$ and $\bar{g}$.

(2) $\rho(\bar{f})$ is either one point or a closed interval.

(3) For any $\alpha \in \rho(\bar{f})$, there exists $x \in R$ such that $\lim _{n \rightarrow \infty}\left(f^{n}(x)-x\right) / n=\alpha$. (1) is trivial from the definition. See [2] and [3] for (2), (3) and other elementary properties of $\rho(\bar{f})$. By (2) we may denote the upper and lower endpoints of $\rho(\bar{f})$ by $\rho_{+}(\bar{f})$ and $\rho_{-}(\bar{f})$ respectively.

The aim of this paper is to prove the following

THEOREM 1. Let $M$ be the set of probability measures on $S$ invariant with respect to $f$. Let $\varphi=\bar{f}-\mathrm{Id}: S \rightarrow R$ where Id: $R \rightarrow R$ denotes identity. Then $\rho(\bar{f})=\{\mu(\varphi) \mid \mu \in$ $M\}$.

Received by the editors November 8, 1982.

1980 Mathematics Subject Classification. Primary 58F20.

Key words and phrases. Rotation set.

1983 American Mathematical Society $0002-9939 / 83 \$ 1.00+\$ .25$ per page 
Notes. (i) The set of probability measures on $S$ is regarded as the set of positive linear functionals $\mu$ on $C(S)$ such that $\mu(1)=1$.

(ii) $S$ is considered to be $[0,1]$ with 0 and 1 identified, and $\varphi(x)=\bar{f}(x)-x$ for $x \in[0,1]$.

THEOREM 2. If $\rho_{+}(\bar{f})\left(\rho_{-}(\bar{f})\right)$ is irrational and $\theta>0$, then $\rho_{+}\left(R_{\theta} \bar{f}\right)>\rho_{+}(\bar{f})$ $\left(\rho_{-}\left(R_{\theta} \bar{f}\right)>\rho_{-}(\bar{f})\right)$ where $R_{\theta}: R \rightarrow R$ is defined as $R_{\theta}(x)=x+\theta$.

These two theorems are generalizations of properties given in Herman [1] for the case $f$ is a homemorphism of $S$.

Considering (1) and Theorem 2, we obtain the following

COROLLARY. If $f$ is structurally stable, then both $\rho_{+}(\bar{f})$ and $\rho_{-}(\bar{f})$ are rational.

Proof of Theorem 1. By (3) above, for any $\alpha \in \rho(f)$, there exists $x \in S$ satisfying

$$
\lim _{n \rightarrow \infty} \frac{1}{n} \sum_{i=0}^{n-1} \varphi\left(f^{i}(x)\right)=\lim _{n \rightarrow x} \frac{1}{n}\left(\bar{f}^{n}(x)-x\right)=\alpha
$$

where $\varphi=\bar{f}-$ Id.

Define $\mu_{n}: C(S) \rightarrow R$ by $\mu_{n}(g)=n^{-1} \sum_{i=0}^{n-1} g\left(f^{i}(x)\right)$ for $g \in C(S)$ using $x$ above. Then $\mu_{n}$ is a probability measure on $S$. Since the set of probability measures is weak* compact, there is a subsequence $\left\{\mu_{k_{n}}\right\}$ of $\left\{\mu_{n}\right\}$ which converges weakly to a probability measure, say $\mu$. That is, we have $\mu_{k_{n}}(g) \rightarrow \mu(g)$ for any $g \in C(S)$. Taking $\varphi=f-$ Id for $g$, we have $\mu_{k_{n}}(\varphi) \rightarrow \mu(\varphi)$, while by definition,

$$
\lim _{n \rightarrow \infty} \mu_{k_{n}}(\varphi)=\lim _{n \rightarrow \infty} \frac{1}{k_{n}} \sum_{i=0}^{k_{n}-1} \varphi\left(f^{i}(x)\right)=\alpha .
$$

Therefore, we have $\mu(\varphi)=\alpha$. Since $\lim _{n \rightarrow x} \mu_{k_{n}}(g)=\mu(g), \mu$ is invariant with respect to $f$.

Consequently $\rho(\bar{f}) \subset\{\mu(\varphi) \mid \mu \in M\}$. On the other hand, for any rational $p / q<$ $\rho_{-}(f)$, we have $\bar{f}^{q}(x)-x-p>0$ for any $x \in S$. Thus $\mu\left(\bar{f}^{q}-\mathrm{Id}-q \cdot p / q\right)>0$ for any $\mu \in M$, while

$$
\mu\left(\bar{f}^{q}-\mathrm{Id}-q \mu(\varphi)\right)=\mu\left(\sum_{i=0}^{q-1}(\bar{f}-\mathrm{Id}) \circ f^{i}\right)-q \mu(\varphi)=0 .
$$

Therefore $\mu(\varphi)>p / q$.

By the same reasoning, we have $\mu(\varphi)<p / q$ for any rational $p / q>\rho_{+}(f)$. Since $\rho(\bar{f})$ is a closed set and $\rho(\bar{f}) \subset\{\mu(\varphi) \mid \mu \in M\}$, we have $\rho(\bar{f})=\{\mu(\varphi) \mid \mu \in M\}$.

We need two lemmas to prove Theorem 2.

LEMMA 1. For any irrational number $\alpha$ there exists a monotone decreasing sequence $\left\{p_{n} / q_{n}\right\}$ (and a monotone increasing sequence $\left\{p_{n}^{\prime} / q_{n}^{\prime}\right\}$ ) of rationals converging to $\alpha$ and satisfying $p_{n} / q_{n}-\alpha<1 / q_{n}^{2}\left(\alpha-p_{n}^{\prime} / q_{n}^{\prime}<1 / q_{n}^{2}\right)$.

As this is a well-known fact in arithmetic, we do not give the proof. 
Lemma 2. Let $\theta>0$. For any $k \in N$ and any $x \in R$, there exists $y \in R$ such that $y \leqslant x$ and $\left(R_{\theta} \bar{f}\right)^{h}(y) \geqslant \bar{f}^{h}(x)+\theta$.

Proof. We prove this by induction. For $k=1$ it is trivial. Assume the lemma is true for $k$, then we have $y \leqslant x$ and $\left(R_{\theta} \bar{f}\right)^{k}(y) \geqslant \bar{f}^{k}(x)+\theta$. Since $\left(R_{\theta} \bar{f}\right)^{k}(s+n)=$ $\left(R_{\theta} \bar{f}\right)^{k}(s)+n$ for $n \in Z$, we have $z \leqslant y$ such that $\left(R_{\theta} \bar{f}\right)^{k}(z)=\bar{f}^{k}(x)$, then

$$
\begin{aligned}
\left(R_{\theta} \bar{f}\right)^{k+1}(z) & =\bar{f}\left(\left(R_{\theta} \bar{f}\right)^{k}(z)\right)+\theta \\
& =\bar{f}\left(\bar{f}^{k}(x)\right)+\theta=\bar{f}^{k+1}(x)+\theta
\end{aligned}
$$

and $z \leqslant x$, completing the induction.

Proof of THEOREM 2. We prove the theorem for $\rho_{+}(\bar{f})$ because the $\rho_{-}(\bar{f})$ case is similar. By Lemma 1, we may choose a sequence $\left\{p_{n} / q_{n}\right\}$ of rationals such that $\left\{p_{n} / q_{n}\right\} \downarrow \alpha$ and $p_{n} / q_{n}-1 / q_{n}^{2}<\alpha$. Since $\rho_{+}(\bar{f})=\alpha$, we have $\bar{f}^{q_{n}}(x)-x<p_{n}$ for any $x \in R$. Suppose that there exists $\theta>0$ such that $\bar{f}^{q_{n}}(x)-x<p_{n}-\theta$ for any $n \in N$ and any $x \in R$. Take $q_{n}$ large enough to satisfy $q_{n} \theta>1$, then we have

$$
\bar{f}^{q_{n}^{2}}(x)-x=\sum_{i=0}^{q_{n}-1}\left\{\bar{f}^{q_{n}}\left(\bar{f}^{i q_{n}}(x)\right)-\bar{f}^{i q_{n}}(x)\right\}<q_{n}\left(p_{n}-\theta\right)<q_{n} p_{n}-1 .
$$

Thus, we have $\rho_{+}(\bar{f})<\left(q_{n} p_{n}-1\right) / q_{n}^{2}<\alpha$, contradicting $\rho_{+}(\bar{f})=\alpha$. Therefore, for any $\theta>0$, there exist $n \in N$ and $x \in R$ such that $\bar{f}^{q_{n}}(x)-x \geqslant p_{n}-\theta$. On the other hand, by Lemma 2 , there exists $y \leqslant x$ satisfying $\left(R_{\theta} \bar{f}\right)^{q_{n}}(y) \geqslant \bar{f}^{q_{n}}(x)+\theta$. Thus, we have $\left(R_{\theta} \bar{f}\right)^{q_{n}}(y)-y>\bar{f}^{q_{n}}(x)-x+\theta \geqslant p_{n}$. Therefore, $\rho_{+}\left(R_{\theta} \bar{f}\right) \geqslant$ $p_{n} / q_{n}>\alpha$, completing the proof.

\section{REFERENCES}

1. M. Herman. Sur la conjugaison différentiable des difféomorphismes du cercle à des rotations, Inst. Hautes Études Sci. Publ. Math. 49 (1979).

2. R. Ito, Rotation sets are closed, Math. Proc. Cambridge Philos Soc. 89 (1981), 107-111.

3. S. Newhouse. J. Palis and F. Takens, Stable families of dinamical sistem. I: diffeomorphisms. I.M.P.A., Rio de Janeiro, Brazil, 1979, preprint.

Department of Mathematics, School of Education. Waseda University, Shinjuku, Tokyo. JAPAN 